\title{
ESPECTROSCOPIA MÖSSBAUER DE VELOCIDAD CERO Y APLICACIONES
}

\author{
Jorge A. Bravo, Yezeña Huaypar, y Mirian E. Mejia \\ Laboratorio de Análisis de Suelos, Facultad de Ciencias Físicas, Universidad Nacional Mayor de San Marcos \\ Apartado Postal 0149, Lima 14, Perú
}

\begin{abstract}
Resumen
Se presenta la base teórica que sustenta la denominada espectroscopia Mössbauer de velocidad cero para medir el espesor resonante nuclear a velocidad cero y el área total para absorción resonante nuclear de una muestra dada en la modalidad de transmisión. Se presenta la metodología para tomar y analizar los datos experimentales, incluyendo parámetros instrumentales y la incertidumbre en los resultados, utilizando señales de forma triangular y sinusoidal.
\end{abstract}

Palabras claves: Resonante nuclear, velocidad cero, absorción resonante nuclear.

\begin{abstract}
The theoretical fundamentals of the so called zero velocity Mössbauer spectroscopy is presented to measure the nuclear resonance thickness at zero velocity and total area for nuclear resonance absorption of a given sample in the transmission mode. The methodology to take and analyze the experimental data is presented, including instrumental parameters and the uncertainty in the results, using triangular and sinusoidal signals.
\end{abstract}

Keywords: Nuclear resonance, zero velocity, nuclear resonance absorption.

\section{Introducción}

La espectroscopia Mössbauer de velocidad cero es una técnica conocida desde el inicio de las aplicaciones del efecto Mössbauer ${ }^{1}$. Sin embargo, a pesar de ser de fácil aplicación, no se la aprovecha como una técnica sencilla y rápida para diagnosticar la presencia y sus propiedades de absorción resonante de nucleidos Mössbauer en una muestra. El propósito de este trabajo es motivar un mayor uso de esta técnica con la presentación de sus fundamentos y el análisis de los datos que se obtienen. Asimismo, se enfatiza la necesidad de conocer los parámetros de operación instrumental ${ }^{2}$ que caracterizan el espectrómetro para poder realizar cálculos cuantitativos. Como se verá, esta técnica permite medir el espesor óptico nuclear resonante en la energía central, es decir a velocidad cero, de la línea de emisión gamma de la fuente Mössbauer y el área total de absorción nuclear resonante de una muestra. Estos datos son valiosos para hacer un diagnóstico temprano de las propiedades resonantes de una muestra. Adicionalmente, es útil para monitorear cambios de fase $^{3}$ en una muestra en función de la temperatura cuando estos cambios de fase están acompañados de cambios bruscos en dichas propiedades resonantes. Como una aplicación adicional y utilizando una muestra patrón como referencia, esta técnica permite diagnosticar el movimiento de la muestra debido a vibraciones producidas por un agente externo. También se incluye un análisis de la incertidumbre en los resultados utilizando el método convencional.

Este análisis es importante para determinar el diseño experimental más adecuado. 


\section{Teoría}

\subsection{Análisis de la radiación $\gamma$ de 14,41 KeV}

En este trabajo nos referiremos a la transición Mössbauer $\gamma 14,41 \mathrm{KeV}$ del nucleido ${ }^{57} \mathrm{Fe}$ y a una fuente Mössbauer de ${ }^{57} \mathrm{Co}$ en una matriz de Rh. La radiación proveniente de esta transición contiene dos componentes: una componente se refiere a la que resulta de las transiciones Mössbauer o "sin rechazo" y la segunda, se refiere a la que resulta de transiciones "con rechazo". Suponemos que $\mathrm{I}_{\mathrm{o}}$ es el total de $\gamma 14,41$ $\mathrm{KeV}$ que son emitidos por segundo hacia el detector de la cadena de espectrometría gamma. La distribución espectral de esta radiación viene dada por

$\mathrm{I}(\mathrm{E}) \mathrm{dE}=\mathrm{f}_{\mathrm{F}} \mathrm{I}_{\mathrm{o}} \mathrm{P}_{\mathrm{M}}\left(\mathrm{E}, \mathrm{E}_{0}\right) \mathrm{dE}+\left(1-\mathrm{f}_{\mathrm{F}}\right) \mathrm{I}_{\mathrm{o}} \mathrm{P}_{\mathrm{D}}\left(\mathrm{E}, \mathrm{E}_{0}{ }^{\prime}\right) \mathrm{dE}$

donde:

$$
P_{M}\left(E, E_{0}\right) d E=\frac{\left(\frac{2}{\Gamma_{F}}\right) d E}{\pi\left[\left(\frac{E-E_{o}}{\Gamma_{F} / 2}\right)^{2}+1\right]},
$$

tal que

$$
\int_{0}^{\infty} P_{M}\left(E, E_{0}\right) d E=1
$$

= distribución espectral de la radiación Mössbauer,

$\Gamma_{\mathrm{F}}=$ ancho de la línea de la fuente $\approx 4,8 \times 10^{-9} \mathrm{eV}$,

$\mathrm{E}_{\mathrm{o}}=$ energía de resonancia o central de la línea,

$$
P_{D}\left(E, E_{0}{ }^{\prime}\right) d E=\frac{\exp \left[-\frac{\left(E-E_{O}^{\prime}\right)^{2}}{2 S^{2}}\right]}{\sqrt{2 \pi s^{2}}} d E,
$$

tal que; $\quad \int_{0}^{\infty}{ }^{P_{D}\left(E, E_{0}\right) d E=1}$

= distribución espectral de la radiación emitida con rechazo, $\mathrm{E}_{0}{ }^{\prime}=\mathrm{E}_{0}-\mathrm{R}$, donde $\mathrm{R}$ es la energía de rechazo, $\mathrm{R}=1,96 \times 10^{-3} \mathrm{eV}$,

$\mathrm{S}^{2}=\Gamma_{\mathrm{D}}^{2} /(8 \ln 2)$, donde $\Gamma_{D}^{2}=4 \ln 2\left(v_{o} / c\right)^{2} E_{o}^{2}$, $\mathrm{v}_{\mathrm{o}}=(2 \mathrm{kT} / \mathrm{m})^{1 / 2}:$ rapidez más probable de los átomos de ${ }^{57} \mathrm{Fe}$,

$\mathrm{T}=$ temperatura $(\mathrm{K})$ de la fuente $\mathrm{y}$

$\Gamma_{\mathrm{D}}=2,33 \times 10^{-2} \mathrm{eV}$ a $293 \mathrm{~K}$.

El valor de $\mathrm{I}_{0}$ está relacionado con la actividad de la fuente y a la geometría del arreglo experimental. Consideraremos que el arreglo corresponde al de transmisión.

Como se puede observar $\Gamma_{\mathrm{F}} / \Gamma_{\mathrm{D}} \approx 2 \times 10^{-7}$; por la tanto, la distribución $\mathrm{P}_{\mathrm{F}}(\mathrm{E})$ es como 5 millones de veces de mayor amplitud y más angosta que $\mathrm{P}_{\mathrm{D}}(\mathrm{E})$. Este hecho jugará un rol muy importante en el análisis de los datos experimentales. $\Gamma_{\mathrm{F}}$ equivale a un corrimiento Doppler de $\approx 0,1$ $\mathrm{mm} / \mathrm{s}$ que se puede comparar con la rápidez más probable de $291 \mathrm{~m} / \mathrm{s}$ de los átomos de ${ }^{57} \mathrm{Fe}$ en un material a temperatura del medio ambiente. Para tomar los espectros Mössbauer normalmente se utiliza rapideces que no superan los $12 \mathrm{~mm} / \mathrm{s}$; esto implica que la modulación en velocidad afecta fuertemente la absorción resonante por los núcleos Mössbauer en la muestra de la componente Mössbauer de la radiación mas no a la absorción resonante de la componente con rechazo, la cual se puede despreciar relativo a la primera.

\subsection{Análisis del espesor óptica de la muestra}

Inicialmente supondremos que la muestra posee una sola resonancia nuclear ubicada en $\mathrm{E}=\mathrm{E}_{1}$, ancho $\Gamma_{\mathrm{A}}$ y espesor óptico para absorción gamma nuclear resonante $\tau_{\mathrm{n}}(\mathrm{E})$. Además la muestra posee espesor óptico para absorción electrónica $\tau_{\mathrm{e}}(\mathrm{E})$. De manera que el espesor óptico total, $\tau(\mathrm{E})$, para absorción de la muestra viene dado por

$$
\tau(\mathrm{E})=\tau_{\mathrm{n}}(\mathrm{E})+\tau_{\mathrm{e}}(\mathrm{E})
$$

donde:

$$
\begin{aligned}
& \tau_{n}\left(E, E_{1}\right)=\frac{\tau_{o}\left(\frac{\Gamma_{n}}{\Gamma_{a}}\right)}{\left(\frac{E-E_{1}}{\Gamma_{a} / 2}\right)^{2}+1}, \\
& \tau_{\mathrm{o}}=\mathrm{f}_{\mathrm{a}} \mathrm{n}_{\mathrm{m}} \sigma_{\mathrm{o}} \delta
\end{aligned}
$$

donde $\tau_{\mathrm{o}}$ es el espesor óptico para absorción resonante en el centro de la línea, $\mathrm{f}_{\mathrm{A}}$ el factor Mössbauer del absorbente, $\mathrm{n}_{\mathrm{m}}$ la densidad de átomos de ${ }^{57} \mathrm{Fe}, \sigma_{\mathrm{o}}$ la sección trasversal para absorción resonante e igual a $2,56 \times 10^{-18} \mathrm{~cm}^{2}$ y $\delta$ es el espesor de la muestra absorbente. Por su lado, $\tau_{\mathrm{e}}(\mathrm{E})$ es una función de variación lenta en 
energía comparado a $\tau_{\mathrm{n}}(\mathrm{E})$ y supondremos que es igual a $\tau_{\mathrm{e} 1}=\tau_{\mathrm{e}}\left(\mathrm{E}_{1}\right)$.

\subsection{Análisis del conteo de radiación $\gamma 14,41 \mathrm{KeV}$ con la fuente en reposo}

El conteo que registra la cadena de espectroscopia gamma se hace con un discriminador de altura de pulsos con una ventana centrada alrededor del pico asociado a $\gamma 14,41 \mathrm{KeV}$. Cuando la fuente Mössbauer se encuentra en reposo, el conteo viene dado por:

$\mathrm{C}_{o}=t_{a} \varepsilon_{1} \exp \left(-\tau_{e l}\right) \int_{0}^{\infty} I\left(E, E_{0}\right) \exp \left(-\tau_{n}\left(E, E_{1}\right)\right) d E+C_{B}$

donde $t_{a}$ es el tiempo de acumulación del espectro; $\varepsilon_{1}=\varepsilon\left(\mathrm{E}_{1}\right)$, expresa la eficiencia de la cadena gamma para contar fotones de energía $\mathrm{E}_{1} ; \tau_{\mathrm{e} 1}=\tau_{\mathrm{e}}\left(\mathrm{E}_{1}\right)$, toma en cuenta que el espesor óptico electrónico es una constante alrededor de $\mathrm{E}_{1}$, el centro de la línea de absorción nuclear resonante; $\mathrm{y}_{\mathrm{B}}$ es el conteo de fondo. Cuando el espesor óptico nuclear de la muestra cumple la condición $\tau_{\mathrm{n}}(\mathrm{E}) \ll<1$, la función exponencial se puede aproximar por los dos primeros términos de su representación en una sucesión de Taylor. Tenemos:

$$
C_{0}=t_{a} \varepsilon_{1} \exp \left(-\tau_{e l}\right)\left[\int_{0}^{\infty} I\left(E, E_{0}\right) d E-\int_{0}^{\infty} \tau_{n}\left(E, E_{1}\right) I\left(E, E_{0}\right) d E\right]+C_{B}
$$

Luego reemplazamos la Ec. (1) en la Ec. (8) y obtenemos:

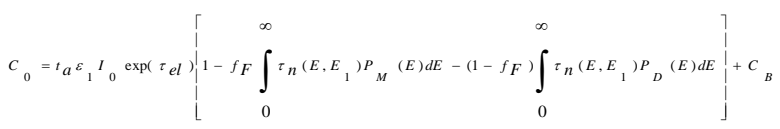

Luego de sustituir las dependencias funcionales de $\tau_{\mathrm{n}}(\mathrm{E}), \mathrm{P}_{\mathrm{M}}(\mathrm{E})$ y $\mathrm{P}_{\mathrm{D}}(\mathrm{E})$, según las Ecs. (2), (3) y (5), tenemos

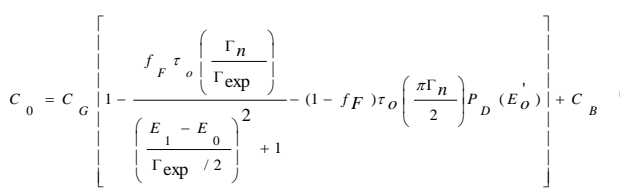

donde $\mathrm{C}_{\mathrm{G}}=\mathrm{t}_{\mathrm{a}} \varepsilon_{1} \mathrm{I}_{\mathrm{o}} \exp \left(-\tau_{\mathrm{el}}\right)$ y $\Gamma_{\text {exp }}=\Gamma_{A}+\Gamma_{F}$.
En el cálculo de la segunda integral se ha aprovechado el hecho que la línea Mössbauer es mucho más angosta comparado a la componente Doppler y se comporta como una función delta de Dirac; dando como resultado un término que se puede despreciar sobre la base que es proporcional a $\left(\Gamma_{\mathrm{n}} / \Gamma_{\mathrm{D}}\right)$ mientras que el segundo término es proporcional a $\left(\Gamma_{\mathrm{n}} / \Gamma_{\text {exp }}\right)$, es decir, del orden de un millón de veces mayor. Luego:

$$
C_{0}=C_{G}\left\lfloor 1-\frac{f_{F} \tau_{o}\left(\frac{\Gamma_{n}}{\Gamma_{\text {exp }}}\right)}{\left.\mid \frac{E_{1}-E_{0}}{\Gamma_{\text {exp }} / 2}\right)^{2}+1}\right\rfloor+C_{B}
$$

Que se puede escribir de una manera más compacta como:

$$
C_{0}=C_{G}\left[1-f_{F} \tau\left(E_{0}, E_{1}\right)\left(\frac{\Gamma_{n}}{\Gamma_{\text {exp }}}\right)\right]+C_{B}
$$

\subsection{Análisis del conteo de radiación $\gamma 14,41 \mathrm{KeV}$ con la fuente en movimiento}

Cuando se utiliza un transductor para mover la fuente de ${ }^{57} \mathrm{Co}$ de acuerdo a una señal periódica en tiempo, es imprescindible tomar en cuenta que la radiación gamma que se emite sufre un corrimiento Doppler dado por:

$$
\mathrm{E}^{\prime}(\mathrm{t})-\mathrm{E}=\mathrm{Ev}(\mathrm{t}) / \mathrm{c}, \quad 0<\mathrm{t}<\mathrm{T}
$$

donde E es la energía del rayo gamma cuando la fuente está en reposo, v (t) es la velocidad de la fuente, $\mathrm{c}$ la rapidez de la luz y $\mathrm{T}$ es el periodo del movimiento. Para la dependencia temporal de $\mathrm{v}$ (t) las modalidades más frecuentes son la de aceleración constante que permite una dependencia lineal de la velocidad en el intervalo de tiempo $\left[-\mathrm{v}_{\mathrm{o}}, \mathrm{v}_{\mathrm{o}}\right]$, y la sinusoidal que permite una variación sinusoidal en el intervalo $\left[-\mathrm{v}_{\mathrm{o}}, \mathrm{v}_{\mathrm{o}}\right]$. Por lo tanto, el tiempo de acumulación del espectro se puede considerar que está constituido de un gran número de intervalos de duración T. El conteo acumulado viene dado por

$C_{1}=t_{a} \varepsilon \exp \left(-\tau_{e}\right) \int_{0}^{T} \frac{d t}{T}\left[1-\int_{0}^{\infty} \tau\left(E, E_{1}\right) I\left[E, E_{0}(t)\right]\right]+C_{B}$ 
donde $E_{0}^{\prime}(t)$, la energía de la transición Mössbauer, es una función del tiempo de acuerdo a la Ec. (13), con $\mathrm{E}_{0}$ en lugar de E. Luego, utilizando la relación Doppler expresada por la Ec. (13), introducimos un cambio de la variable tiempo a la variable energía dada por

$$
\frac{d v}{c}=\frac{a(t) d t}{c}=\frac{d E_{0}^{\prime}}{E_{0}}
$$

Luego de despejar dt en esta relación y sustituir en la Ec. (15) obtenemos

$$
C_{1}=t_{a} \varepsilon \exp \left(-\tau_{e}\right) \int_{E_{o}-\delta E}^{E_{0}+\delta E} \frac{c d E_{0}}{a\left(E_{0}\right) T E_{0}}\left[I_{o}-\int_{0}^{\infty} \tau\left(E, E_{1}\right) I\left(E, E_{0}\right) d E\right]+C_{B}
$$

donde $\delta \mathrm{E}=\frac{v_{o}}{c} E_{0}$. La integración sobre la variable $\mathrm{E}$ se puede realizar de inmediato $\mathrm{y}$ obtenemos:

$$
\left.C_{1=C_{G}} \int_{E_{o}-\delta E}^{E_{0}+\delta E} \frac{c d E_{0}^{\prime}}{a\left(E_{0}\right) T E}\right|_{[}\left|-f_{F} \frac{\tau_{o}\left(\frac{\Gamma_{n}}{\Gamma_{\text {exp }}}\right)}{\left(\frac{E_{1}-E_{o}^{\prime}}{\Gamma_{\text {exp }} / 2}\right)^{2}+1}-\left(1-f_{F}\right) \tau_{o}\left(\frac{\pi \Gamma_{n}}{2}\right) P_{D}\left(E_{1}\right)\right|+C_{B}
$$

Por las razones ya expuestas, el tercer término de la derecha de esta ecuación puede ser despreciado por ser del orden de un millón de veces más pequeño que las otras dos. En los casos de interés, el valor de $\mathrm{v}_{\mathrm{o}}$ se escoge de tal manera que el intervalo de energías $\left(\mathrm{E}_{0}-\delta \mathrm{E}, \mathrm{E}_{0}\right.$ $+\delta \mathrm{E}$ ) incluye todas las resonancias que posee la muestra absorbente

\subsubsection{Modalidad de Aceleración Constante}

En este caso, por ser la aceleración constante, tenemos que: $\quad \mathrm{a}\left(\mathrm{E}_{0}{ }^{\prime}\right) \mathrm{TE}_{0} / \mathrm{c}=\mathrm{a}_{0} \mathrm{TE}_{0} / \mathrm{c}=2 \mathrm{v}_{0} \mathrm{E}_{0} / \mathrm{c}$ $=2 \delta \mathrm{E}_{1}$, y las integraciones se pueden realizar $\sin$ mayor dificultad para obtener:

$$
C_{1}=C_{G}\left\lceil 1-\frac{\text { Area }}{2 \delta E_{1}}\right]+C_{B},
$$

donde $\quad$ Area $=f_{F} \tau \sigma_{o} \pi \Gamma_{n} / 2$
Se observa que el segundo término de la derecha es inversamente proporcional a $\delta \mathrm{E}_{1}$ mientras que en el caso de la Ec. (12) es inversamente proporcional a $\Gamma_{\text {exp. }}$. Si consideramos valores típicos como $\delta \mathrm{E}_{1}=10 \mathrm{~mm} / \mathrm{s}$ y $\Gamma_{\exp }=0,5 \mathrm{~mm} / \mathrm{s}$, vemos que el segundo término de la Ec. (18) es como 25 veces más pequeño que en el caso de la Ec. (12).

\subsubsection{Modalidad de velocidad con variación sinusoidal}

En este caso consideramos que la velocidad viene dada por:

$\mathrm{v}(\mathrm{t})=-\mathrm{v}_{\mathrm{o}} \cos \omega \mathrm{t}, \operatorname{con} 0<\mathrm{t}<\mathrm{T}, \omega=\pi / \mathrm{T}$

Además, tenemos la siguiente relación para el corrimiento Doppler:

$$
\frac{v(t)}{v_{o}}=\frac{c\left[E_{0}(t)-E_{0}\right]}{v_{0} E_{0}}
$$

Que permite realizar un cambio de variable de $E_{o}{ }^{\prime}(t)$ a $v(t)$. La Ec. (17) toma la siguiente forma:

$C_{1}=C_{G} \int_{-v_{o}}^{v_{o}} \frac{d v}{\pi v_{o} \sqrt{1-\left(v / v_{o}\right)^{2}}}\left|-f_{F} \frac{\tau_{o}\left(\frac{\Gamma_{n}}{\Gamma_{\exp }}\right)}{\left(\frac{E_{1}-E_{o}(v)}{\Gamma_{\text {exp }} / 2}\right)^{2}+1}\right|+C_{B}$

El cálculo exacto del segundo término de esta integral requiere la aplicación de un método de integración numérica. Un valor aproximado se puede obtener si consideramos que la energía de resonancia yace cerca de $\mathrm{E}_{0}$ y lejos de los extremos del intervalo de integración; en tal caso el radical en el denominador del integrando se puede tomar como unidad y obtenemos:

$$
C_{1} \approx C_{G}\left[1-\frac{\text { Area }}{\pi \delta E_{1}}\right]+C_{B},
$$

En este caso, el valor de la integral es ligeramente mayor que en el caso de aceleración constante. 


\subsection{Generalización para el caso de múltiples resonancias}

Consideramos el caso en que el absorbente posee un espesor óptico resonante dado por $\tau_{\mathrm{n}}(\mathrm{E})$. Generalmente los anchos de línea de las resonancias son mayores que $\Gamma_{\mathrm{F}}$, lo cual permite considerar que $\tau_{\mathrm{n}}(\mathrm{E})$ es una función de variación lenta comparado a $\mathrm{P}_{\mathrm{M}}\left(\mathrm{E}, \mathrm{E}_{0}\right)$. Tomando como referencia los resultados obtenidos para una sola resonancia es posible generalizar dichos resultados para el caso en que existen varias resonancias que pueden superponerse entre si. Tomando este hecho como referencia, podemos considerar que $\mathrm{P}_{\mathrm{M}}\left(\mathrm{E}, \mathrm{E}_{0}\right)$ se comporta como una función delta de Dirac. De manera que cuando la fuente se encuentra en reposo, la Ec. (12) se puede generalizar en la forma siguiente:

$$
C_{o}=C_{G}\left[1-f_{F} \tau_{n}\left(E_{0}\right)\right]+C_{B}
$$

Cuando la fuente se mueve con aceleración constante y el corrimiento Doppler incluye todas las resonancias del absorbente, la Ec. (18) se puede generalizar en la forma siguiente:

$C_{1}=C_{G}\left\lceil 1-\left(\frac{\text { Area }}{2 \delta E_{1}}\right)\right\rceil+C_{B}$,

donde ${ }^{\text {Area }}=f_{F} \int_{E_{0}-\delta E}^{E_{0}+\delta E} \tau_{n}(E) d E$ es el área total de

absorción nuclear resonante de la muestra, que es un parámetro relacionado con el área total no corregida, $\mathrm{C}_{\mathrm{G}}$ Area, de absorción nuclear resonante que calculan los programas de ajuste de espectros; $\delta \mathrm{E}_{1}$ está asociado a la rapidez máxima que alcanza la fuente. Nuevamente, en los casos en que el espesor óptico de la muestra a velocidad cero sea significativo, esperamos que $\mathrm{C}_{1}$ sea mayor que $\mathrm{C}_{\mathrm{o}}$ ya que $\delta \mathrm{E}_{1}$ es mayor que el rango de energías asociado a las resonancias. Si la muestra es ópticamente gruesa para absorción gamma nuclear el Área viene dada por:

$$
\text { Area }=f_{F} \int_{E_{0}-\delta E}^{E_{0}+\delta E}(1-\exp (-\tau(E)) d E
$$

\subsection{Obtención de los parámetros Mössbauer}

A continuación se presenta un método que permite deducir los parámetros Mössbauer $\tau_{\mathrm{n}}\left(\mathrm{E}_{0}\right)$ y el Área de la muestra a partir de los datos experimentales, con el uso de las Ecs. (23) y (24). El parámetro $f_{F}$ se considera como conocido y es medido normalmente por el fabricante de la fuente; el tiempo de acumulación de las cuentas es el mismo en ambos casos. $\delta \mathrm{E}_{1}$ es un dato experimental conocido; las cantidades $\mathrm{C}_{\mathrm{o}}$ y $\mathrm{C}_{1}$ corresponden al número de cuentas registradas y son datos experimentales. Asimismo, supondremos que se conoce la razón $\alpha=\mathrm{C}_{\mathrm{B}} / \mathrm{C}_{\mathrm{G}}$, que es un parámetro que se puede medir con una muestra patrón. De manera que tenemos sólo 2 ecuaciones para deducir las siguientes cantidades: $\mathrm{C}_{\mathrm{G}}, \tau_{\mathrm{n}}\left(\mathrm{E}_{0}\right)$ y Área. Para obtener una tercera relación es necesario realizar otro conteo con otra velocidad máxima, que supondremos que es mayor que la anterior, que da lugar a un corrimiento Doppler igual a $\delta \mathrm{E}_{2}$. De manera que tenemos las siguientes tres ecuaciones:

$$
\begin{aligned}
& C_{0}=C_{G}\left[(1+\alpha)-f_{F} \tau_{n}\left(E_{0}\right)\right] \\
& C_{1}=C_{G}\left[(1+\alpha)-\left(\frac{\text { Area }}{2 \delta E_{1}}\right)\right] \\
& C_{2}=C_{G}\left[(1+\alpha)-\left(\frac{\text { Area }}{2 \delta E_{2}}\right)\right]
\end{aligned}
$$

Estas tres ecuaciones se pueden combinar de la manera siguiente:

$$
\frac{C_{1}-C_{0}}{C_{2}-C_{0}}=\frac{1-\left(\frac{1}{2 \delta E_{1}}\right) \Gamma_{E}}{1-\left(\frac{1}{2 \delta E_{2}}\right) \Gamma_{E}}=R
$$

donde hemos definido el ancho resonante efectivo de la muestra como

$$
\Gamma_{E}=\frac{\text { Area }}{f_{F} \tau\left(E_{0}\right)}
$$

De la Ec. (26) se puede espejar $\Gamma_{\mathrm{E}}$ : 


$$
\Gamma_{E}=\frac{2(1-R) \delta E_{1}}{1-\frac{R \delta E_{1}}{\delta E_{2}}}
$$

Las Ecs. (25 b) y (25 c) se pueden combinar para obtener la siguiente relación:

$$
\frac{C_{2}-C_{1}}{C_{2}}=\frac{\text { Area }\left(\frac{1}{2 \delta E_{1}}-\frac{1}{2 \delta E_{2}}\right)}{(1+\alpha)-\frac{\text { Area }}{2 \delta E_{2}}}=S
$$

de la cual se puede despejar el Área para obtener:

$$
\text { Area }=\frac{2 \delta E_{1}(1+\alpha) S}{1+(S-1)\left(\frac{\delta E_{1}}{\delta E_{2}}\right)}
$$

De manera que las ecuaciones (27), (28) y (30) expresan la solución del problema. En el caso de una señal sinusoidal será necesario utilizar la relación expresada por la Ec. (22) en lugar de la Ec. (18). Se ha elaborado un programa en lenguaje FORTRAN para hacer los cálculos requeridos incluyendo la incertidumbre en los parámetros calculados.

\subsection{Caso de vibración de la muestra producida por un agente externo}

Consideramos el caso de una muestra acoplada a un agente externo que da lugar a que ésta vibre con frecuencia angular $\omega \mathrm{y}$ con amplitud y velocidad desconocidas, de manera que el desplazamiento de la muestra relativo al detector viene dado por las siguientes ecuaciones:

$X(t)=X_{o} \operatorname{sen} \omega t$

$\mathrm{V}(\mathrm{t})=\mathrm{V}_{1} \cos \omega \mathrm{t}$, con $\mathrm{V}_{1}=\omega \mathrm{X}_{\mathrm{o}}$

$(31 \mathrm{a}, \mathrm{b})$

Para fines de diagnóstico de este movimiento, es necesario medir $\mathrm{V}_{1}$. Con este fin se utiliza como absorbedor una muestra de referencia con propiedades de absorción gamma nuclear resonante conocida y se trata de deducir $\mathrm{V}_{1}$ a partir de tres mediciones tal como se ha explicado en la sección anterior: una con la fuente y el absorbedor en reposo, que da lectura $\mathrm{C}_{\mathrm{o}}$; otra con la fuente en reposo y el absorbedor en vibración, que da la lectura $\mathrm{C}_{1}$, y una tercera con el absorbedor en reposo y la fuente en movimiento con una velocidad de amplitud conocida, que da lectura $\mathrm{C}_{2}$. En el caso que la fuente se mueve a aceleración constante se tiene el siguiente resultado:

$$
\begin{aligned}
& \delta E_{1} \approx \frac{\Gamma_{E} / \pi}{1-R\left(1-\frac{\Gamma_{E}}{2 \delta E_{2}}\right)} \\
& V_{1}=\left(\frac{\delta E_{1}}{E_{0}}\right) c
\end{aligned}
$$

Donde R está definido por

$$
R=\frac{1-\left(\frac{1}{\pi \delta E_{1}}\right) \Gamma_{E}}{1-\left(\frac{1}{2 \delta E_{2}}\right) \Gamma_{E}}
$$

El parámetro $\Gamma_{\mathrm{E}}$ se supone que es un parámetro conocido de la muestra de referencia.

\subsection{Incertidumbre en los resultados}

Como se ha podido apreciar la obtención de los resultados requiere calcular diferencias entre los conteos $\mathrm{C}_{0}, \mathrm{C}_{1}$ y $\mathrm{C}_{2}$, los cuales son números de valor muy próximo. La incertidumbre estadística relativa de la diferencia de dos conteos de valor muy próximo es bastante mayor que la incertidumbre relativa de cada uno de ellos; este efecto se conoce como la pérdida de cifras significativas. La incertidumbre, con un intervalo de confianza de $68 \%$, en el conteo C viene dado por $\mathrm{U}=\mathrm{C}^{1 / 2}$ y la incertidumbre relativa viene dada por $\mathrm{U}_{\mathrm{r}}=\mathrm{C}^{-1 / 2}$. Supongamos que tenemos dos conteos $\mathrm{C}_{0}$ y $\mathrm{C}_{1}$ de valor muy próximo, es decir, que la diferencia dada por $\delta_{10}$ $=\mathrm{C}_{1}-\mathrm{C}_{0}$ es del orden de partes por cien. La incertidumbre de esta diferencia viene dada por

$$
\mathrm{U}_{10}=\left(\mathrm{C}_{0}+\mathrm{C}_{1}\right)^{1 / 2}=(2 \mathrm{C})^{1 / 2}
$$

donde $\mathrm{C}$ es el valor promedio de los conteos y la incertidumbre relativa correspondiente viene dada por

$$
\mathrm{U}_{10 \mathrm{r}}=\mathrm{U}_{10} / \delta_{10}
$$


Tomando como referencia las Ecs. (26) y (29), las incertidumbres $U_{S}$ y $U_{R}$ de los parámetros $S$ y $\mathrm{R}$ respectivamente, vienen dados por las siguientes expresiones:

$$
\begin{aligned}
U_{S} & =U_{C_{1} / C_{2}}=\frac{C_{1}}{C_{2}} \sqrt{\frac{1}{C_{1}}+\frac{1}{C_{2}}} \\
U_{R} & =\frac{\sqrt{U^{2} C_{1} / C_{0}+R^{2} U^{2} C_{2} / C_{0}}}{\frac{C_{2}}{C_{0}}-1}
\end{aligned}
$$

Sobre la base de estas incertidumbres se puede calcular las incertidumbres de los parámetros de interés: $\mathrm{f}_{\mathrm{F}} \tau_{\mathrm{n}}\left(\mathrm{E}_{0}\right)$, Área y $\Gamma_{\mathrm{E}}$, utilizando las ecuaciones desarrolladas y la metodología estadística convencional. Para reducir la incertidumbre en los resultados, debido al conteo, será necesario aumentar los conteos; si se aumenta el conteo en un factor de 4 , por ejemplo, las incertidumbres relativas se reducirán a la mitad del valor de referencia.

Como se desea que el tiempo de acumulación no sea demasiado largo y que estos resultados sirvan de diagnóstico temprano, el nivel del conteo que se desee utilizar no puede ser demasiado alto, probablemente se ubique entre $10^{6}$ y $10^{7}$ cuentas.

Otra alternativa es tomar conteos para un número mayor de valores de la velocidad máxima. Por ejemplo, si se tomara un conteo adicional $\mathrm{C}_{3}$ espaciando los valores de la velocidad máxima, entonces podremos calcular tres conjuntos de parámetros en lugar de uno solo. Esto permitiría reducir la incertidumbre en 1so resultados a casi la mitad. La incertidumbre en los resultados también incluirá las incertidumbres en los parámetros operativos del espectrómetro como tiempo muerto y fracción de conteo de fondo.

\section{Arreglo experimental}

\subsection{Calibración del espectrómetro}

Esta calibración incluye:(a)la graduación de la velocidad máxima, $v_{0}$, que le imprimará el transductor a la fuente, (b)la determinación de la fracción de conteo de fondo y (c) la determinación del tiempo muerto de la cadena de espectrometría gamma. La graduación de $\mathrm{v}_{\mathrm{o}}$ se realiza utilizando el método de calibración de la velocidad usando una muestra patrón de $\mathrm{Fe}$ metálico, lo cual permite graduar el control de velocidad del transductor. La fracción de conteo de fondo, B, es mínimo cuando la ventana del analizador monocanal, que selecciona el pico de $\gamma 14,41 \mathrm{KeV}$, está centrado alrededor de este pico; adicionalmente, se recomienda que el ancho de la ventana sea aproximadamente 1,8 veces el ancho del pico a media altura. Más adelante se explicará el método para determinar B.El tiempo muerto de la cadena de Espectrometría gamma debe ser conocido para poder determinar el conteo de saturación de rayos $\gamma 14,41 \mathrm{KeV}$. Para este fin se puede utilizar el método de dos fuentes.

\subsection{Determinación del espesor óptico no resonante de la muestra}

Se recomienda que el espesor óptico, $\tau\left(\mathrm{E}_{\mathrm{o}}\right)$, de la muestra sea aproximadamente igual al de la muestra patrón de calibración de espesor óptico, que se escoge que tenga un espesor óptico $\tau\left(\mathrm{E}_{\mathrm{o}}\right)$ igual a 1,60. Supongamos que $C_{p}$ y $C_{m}$ sean las tasas de conteo que se registran para el patrón y la muestra de estudio respectivamente. Por lo tanto, tenemos la siguiente relación:

$$
\frac{\exp \left(-\tau\left(E_{o}\right)\right)}{\exp (-1,60)}=\frac{C_{m}}{C_{p}}
$$

De donde podemos despejar el espesor óptico de la muestra y obtenemos:

$\tau_{e}\left(E_{o}\right) \approx \tau\left(E_{o}\right)=1,60-\ln \left(\frac{C_{m}}{C_{p}}\right)$

Teniendo en cuenta que el $\tau_{\mathrm{n}}\left(\mathrm{E}_{\mathrm{o}}\right)<<\tau_{\mathrm{e}}\left(\mathrm{E}_{\mathrm{o}}\right)$. Una vez conocido $\tau_{\mathrm{e}}\left(\mathrm{E}_{\mathrm{o}}\right)$ se puede deducir la razón de conteo de fondo a conteo de $\gamma 14.41 \mathrm{KeV}$, que viene dado por:

$\alpha=\frac{C_{B}}{C_{G}}=\frac{B}{G}=\frac{B_{o}}{G_{o} \exp \left(-\tau_{e}\left(E_{o}\right)\right)}$

Donde $G_{0}$ y $B_{0}$ son las fracciones respectivas cuando no hay muestra presente. Se supone que el parámetro $B_{o}$ no varía apreciablemente la medida que aumenta $\tau_{\mathrm{e}}\left(\mathrm{E}_{\mathrm{o}}\right)$, lo cual no ocurre 
para la fracción de $\gamma 14,41 \mathrm{KeV}$ que es atenuada por la muestra. Como debe cumplirse que $\mathrm{G}+\mathrm{B}$ $=1$, tenemos finalmente:

$$
G=\frac{1}{1+\alpha} \quad y \quad B=\frac{\alpha}{1+\alpha}
$$

Se ha observado que $B_{o}$ es aproximadamente igual 0,05 cuando se toma las debidas precauciones $^{2}$.

\section{Resultados}

A continuación se cita algunos resultados obtenidos con muestras analizadas en el Laboratorio de Espectroscopia Mössbauer de la Facultad de Ciencias Físicas utilizando el programa de cálculo ya mencionado. Como hay interés en comparar los resultados de las mediciones de diagnóstico temprano con los valores obtenidos del ajuste de los espectros Mössbauer respectivos, merece recalcar que el parámetro Área que dan los programas de ajuste corresponde al valor de $\mathrm{G}_{\mathrm{G}}$ Area presentado en este trabajo. Asimismo, la amplitud de la absorción resonante a velocidad cero que se observa en el espectro corresponde al valor de $\mathrm{G}_{\mathrm{G}} \mathrm{f}_{\mathrm{F}} \tau\left(\mathrm{E}_{\mathrm{o}}\right)$ en este trabajo. Merece también mencionar que para un análisis cuantitativo se requiere del parámetro Area $/ f_{F}$, donde $f_{F}$ es un parámetro que mide el fabricante de la fuente de ${ }^{57} \mathrm{Co}$.

Consideramos una muestra de arcilla. Los conteos se realizaron a velocidades de $0 \mathrm{~mm} / \mathrm{s} ; 11,0 \mathrm{~mm} / \mathrm{s}$ y $50,0 \mathrm{~mm} / \mathrm{s}$, durante un intervalo de $265 \mathrm{~s}$ para cada uno, que otorgan como resultado los siguientes conteos:

$$
\begin{aligned}
& \mathrm{C}_{0}=1310073 \mathrm{cts} ; \\
& \mathrm{C}_{1}=1367658 \mathrm{cts} ; \quad \mathrm{y} \\
& \mathrm{C}_{2}=1376858 \mathrm{cts}
\end{aligned}
$$

El espesor óptico no resonante de esta muestra se puede estimar comparando el conteo $\mathrm{C}_{3}$ con el conteo a velocidad cero obtenido con un absorbedor que contiene 40 hojas de papel aluminio, cuyo espesor óptico es 1,63, que fue de 2313724 cts para el mismo intervalo de tiempo. El tiempo muerto de la cadena de espectroscopia gamma utilizado es de $50 \mu \mathrm{s}$ aproximadamente y el parámetro $\mathrm{B}_{\mathrm{o}}$ se tomó igual a 0,05 . Los parámetros calculados fueron:

$$
\begin{array}{ll}
\mathrm{G}_{\mathrm{G}} \mathrm{f}_{\mathrm{F}} \tau\left(\mathrm{E}_{\mathrm{o}}\right) & =0,0503 \pm 0,0151 ; \\
\mathrm{G}_{\mathrm{G}} \text { Area } & =(0,2955 \pm 0,0530) \mathrm{mm} / \mathrm{s} \\
\Gamma_{\mathrm{E}} & =5,875 \pm 2,427
\end{array}
$$

Donde las incertidumbres de los parámetros medidos derivan sólo de las incertidumbres en los conteos. Los valores de $\mathrm{G}_{\mathrm{G}} \mathrm{f}_{\mathrm{F}} \tau\left(\mathrm{E}_{\mathrm{o}}\right)$ y $\Gamma_{\mathrm{E}}$ corresponden a una muestra magnética que en velocidad cero absorbe con mediana intensidad. Por otro lado, el ajuste del espectro da como resultado el valor de $\mathrm{G}_{\mathrm{G}}$ Area = $(0,2207 \pm 0,0012) \mathrm{mm} / \mathrm{s}$. Como se puede observar, la incertidumbre en el resultado que da el ajuste del espectro es mucho menor como debe ser, pero que ha tomado muchas horas de acumulación de cuentas. La Tabla 1 muestra los resultados con otras muestras.

Tabla 1. Resultados de la medida de espesores ópticos y áreas de absorción resonante comparado con los resultados del análisis de espectros convencionales.

\begin{tabular}{|c|c|c|c|c|}
\hline & \multicolumn{3}{|c|}{ Por velocidad cero } & Convencional \\
\hline $\begin{array}{c}\text { Muestra/ } \\
\text { Intervalo }\end{array}$ & $\mathrm{f}_{\mathrm{F}} \tau$ & $\mathrm{f}_{\mathrm{F}}$ Area $(\mathrm{mm} / \mathrm{s})$ & $\Gamma_{\mathrm{E}}$ & $\mathrm{f}_{\mathrm{F}}$ Area $(\mathrm{mm} / \mathrm{s})$ \\
\hline $\begin{array}{c}\text { Fe metálico } \\
265 \mathrm{~s}\end{array}$ & $0,0096 \pm 0,0510$ & $0,5455 \pm 0,0646$ & $57,0 \pm 7,6$ & $0,2419 \pm 0,0030$ \\
\hline $\begin{array}{c}\text { NPS } \\
265 \mathrm{~s}\end{array}$ & $0,0064 \pm 0,0012$ & $0,0747 \pm 0,0107$ & $10,1 \pm 3,5$ & $0,0273 \pm 0,0012$ \\
\hline $\begin{array}{c}\text { Arcilla Greda } \\
265 \mathrm{~s}\end{array}$ & $0,0374 \pm 0,0153$ & $0,0697 \pm 0,0170$ & $1,86 \pm 1,38$ & $0,0612 \pm 0,0006$ \\
\hline $\begin{array}{c}\text { Arcilla Ica } \\
530 \mathrm{~s}\end{array}$ & $0,0065 \pm 0,0090$ & $0,0116 \pm 0,0096$ & $1,71 \pm 1,34$ & $0.0132 \pm 0,0011$ \\
\hline
\end{tabular}

Los altos valores del parámetro $\Gamma_{\mathrm{E}}$ en el caso de Fe metálico y NPS se debe que su espesor óptico resonante para velocidad cero es muy pequeño. Las arcillas citadas no poseen componente magnética.

\section{Conclusiones y Recomendaciones}

Los resultados obtenidos muestran que esta técnica puede ser útil para realizar un diagnóstico temprano de las propiedades de absorción resonante de una muestra. Asimismo, se aprecia su potencial para utilizarla para monitorear transiciones de fase durante el tratamiento térmico de un material. Se recomienda montar un sistema que permita realizar estas mediciones se manera autónoma; es decir, sin depender de un espectrómetro Mössbauer convencional. En el caso de la Facultad de Ciencia Físicas de la UNMSM hace 
falta diseñar y construir un transductor con su sistema de control que permita seleccionar las velocidades requeridas. Para completar el sistema se cuenta con un contador y una cadena de espectroscopia gamma.

\section{Reconocimiento}

Los autores agradecen al Laboratorio de Espectroscopia Mössbauer de la Facultad de Ciencias Físicas de la UNMSM por las facilidades prestadas para llevar a cabo las mediciones que se reportan y por haber motivado a desarrollar este trabajo.

\section{Referencias}

[1] R.W. Grant, Mössbauer Spectroscopy, edited by U. Gonser, Topics in Applied Physics, Vol. 5, Springer-Verlag, Berlin (1975).

[2] Bravo J. A., Cerón M.L. y Fabián J. Optimization Criteria in Mössbauer Spectroscopy. Hyperfine Interactions 148/149: p. 253-261 (2005).

[3] N. Lakshire, K. Venugopolan y J. Varma, Hyperfine field studies of $\mathrm{Fe}_{3} \mathrm{Al}$ and $\mathrm{Fe}_{3-\mathrm{x}}$ $\mathrm{Cr}_{\mathrm{x}} \mathrm{Al}$ alloys, Physical Review B, V47, 21. p. 1054-1061. 